\title{
Singularities in Speckled Speckle
}

\author{
Isaac Freund ${ }^{1}$ and David A. Kessler ${ }^{1}$ \\ ${ }^{1}$ Department of Physics, Bar-Ilan University, Ramat-Gan IL52900, ISRAEL
}

Speckle patterns produced by random optical fields with two (or more) widely different correlation lengths exhibit speckle spots that are themselves highly speckled. Using computer simulations and analytic theory we present results for the point singularities of speckled speckle fields: optical vortices in scalar (one polarization component) fields; $\mathrm{C}$ points in vector (two polarization component) fields. In single correlation length fields both types of singularities tend to be more-or-less uniformly distributed. In contrast, the singularity structure of speckled speckle is anomalous: for some sets of source parameters vortices and $\mathrm{C}$ points tend to form widely separated giant clusters, for other parameter sets these singularities tend to form chains that surround large empty regions. The critical point statistics of speckled speckle is also anomalous. In scalar (vector) single correlation length fields phase (azimuthal) extrema are always outnumbered by vortices (C points). In contrast, in speckled speckle fields, phase extrema can outnumber vortices, and azimuthal extrema can outnumber $\mathrm{C}$ points, by factors that can easily exceed $10^{4}$ for experimentally realistic source parameters.

(C) 2021 Optical Society of America

OCIS codes: $030.0030,030.6140,030.6600,260.0260,260.5430,260.5430,290.0290$

Studies of the singularities of random optical fields (speckle patterns) normally involve fields with a single correlation length $\Lambda[1,2,3,4,5,6,7,8,9,10,11,12,13$, 14, 15, 16, 17, 18, 19, 20. Here we study random fields with two widely different $\Lambda$, finding anomalous spatial distributions for the singularities, and anomalous statistics for them and their associated critical points. These interesting anomalies can be controlled by varying source parameters, and although studied here by computer simulation and analytic theory, they should also be amenable to experimental observation.

The presence of two distinct $\Lambda$ in a random optical field is easily determined visually, because the major speckle spots in the speckle pattern are themselves highly speckled, Figs. 1 and 2. We call such patterns "speckled speckle" [21].

Speckled speckle, which arises from source distributions with widely different length scales, is often the norm. It appears in an uncontrolled form in many scattering experiments due to parasitic scattering, but because the detector resolution is often insufficient to resolve the small speckle spots, the presence of speckled speckle may not be noticed. But it makes its presence felt in discrepancies between experiment and theory, and is, for example, the most common cause of the failure to obtain negative exponential statistics for the intensity. Due to interference, parasitic fields with intensities less than $10^{-8}$ of the main beam can produce measurable effects.

Here we consider controlled situations in which the long correlation length $\Lambda_{a}$ and the short correlation length, $\Lambda_{b}$ and their associated intensities $I_{a}$ and $I_{b}$, are chosen to have values that are experimentally realistic.

Commonly used rotationally symmetric (isotropic) source distributions $S\left(r^{\prime}\right)$, and their associated autocorrelation functions $W(r)$, for circular Gaussian fields [1] with a single correlation length are: $(i)$ a Gaussian, $S_{G}\left(r^{\prime}\right)=\exp \left[-r^{\prime 2} /(2 p)^{2}\right], W_{G}(r)=\exp \left(-\kappa^{2} p^{2} r^{2}\right),(i i)$ a disk, $S_{D}\left(r^{\prime}\right)=\Theta\left(r^{\prime}-p\right), W_{D}(r)=2 J_{1}(\kappa p r) /(\kappa p r)$, and (iii) a thin ring, modeled as $S_{R}\left(r^{\prime}\right)=\delta\left(r^{\prime}-p\right)$, $W_{R}(r)=J_{0}(\kappa p r)$. Here $J_{n}$ is a Bessel function of integer order $n$, and $\kappa=2 \pi /(\lambda Z)$, where $\lambda$ is the wavelength of light, and $Z$ is the distance to the screen in the far field on which the speckle pattern is measured [1]. In what follows we set for convenience $\kappa=1$. In each of the above three cases $\Lambda \sim 1 / p$.

Controlled fields with two different correlation lengths and known correlation functions are easily generated using linear combinations of two of the above source functions with different values for the parameter $p$. There are nine such combinations; here we consider the three in which both source functions are the same - two Gaussians, two disks, and two rings. Writing: $(i)$ for the Gaussians, $S_{a}\left(r^{\prime}\right)=\exp \left[-r^{\prime 2} /(2 a)^{2}\right], W_{a}(r)=\exp \left(-a^{2} r^{2}\right)$, $S_{b}\left(r^{\prime}\right)=\exp \left[-r^{\prime 2} /(2 b)^{2}\right], W_{b}(r)=\exp \left(-b^{2} r^{2}\right) ;(i i)$ for the disks, $S_{a}\left(r^{\prime}\right)=\Theta\left(r^{\prime}-a\right), W_{a}(r)=2 J_{1}(a r) /(a r)$, $S_{b}\left(r^{\prime}\right)=\Theta\left(r^{\prime}-b\right), W_{b}(r)=2 J_{1}(b r) /(b r) ;$ and $(i i i)$ for the rings, $S_{a}\left(r^{\prime}\right)=\delta\left(r^{\prime}-a\right), W_{a}(r)=J_{0}(a r)$, $S_{b}\left(r^{\prime}\right)=\delta\left(r^{\prime}-b\right), W_{b}(r)=J_{0}(b r)$; we have for our compound source function $S_{a b}\left(r^{\prime}\right)$ and its scalar far-field autocorrelation function $W_{a b}(r)$,

$$
\begin{aligned}
S_{a b}\left(r^{\prime}\right) & =I_{a} S_{a}\left(r^{\prime}\right)+I_{b} S_{b}\left(r^{\prime}\right) \\
W_{a b}(r) & =\left[W_{a}(r)+K W_{b}(r)\right] /(1+K),
\end{aligned}
$$


where for the Gaussians and the disks $K=b^{2} I_{b} /\left(a^{2} I_{a}\right)$, and for rings $K=b I_{b} /\left(a I_{a}\right)$. Here $\Lambda_{a} \sim 1 / a, \Lambda_{b} \sim 1 / b$.

The point singularities of generic scalar (one polarization component) fields are optical vortices [2, 22, whereas for generic vector (two polarization component) fields the point singularities are $\mathrm{C}$ points - isolated points of circular polarization embedded in a field of elliptical polarization [12, 13, 22]. It is convenient to treat C points as vortices of the complex Stokes field $S_{12}=$ $S_{1}+i S_{2}$ 23, 24, where in terms of Cartesian field components $E_{x}$ and $E_{y}$, and right (R) and left (L) circularly polarized components, $E_{R}$ and $E_{L}$,

$$
\begin{aligned}
& S_{1}=\left|E_{x}\right|^{2}-\left|E_{y}\right|^{2}=2 \operatorname{Re}\left(E_{R}^{*} E_{L}\right), \\
& S_{2}=2 \operatorname{Re}\left(E_{x}^{*} E_{y}\right)=2 \operatorname{Im}\left(E_{R}^{*} E_{L}\right) .
\end{aligned}
$$

Here Cartesian and circular field components are related by

$$
E_{R}=\left(E_{x}-i E_{y}\right) / 2^{1 / 2}, \quad E_{L}=\left(E_{x}+i E_{y}\right) / 2^{1 / 2}
$$

C points are also optical vortices of $E_{R}$ and of $E_{L}$, because at a point where one of these fields vanishes, as happens at an optical vortex, the optical polarization is circular, whereas elsewhere in the field when both components are nonzero the polarization is in general elliptical. Thus the $\mathrm{C}$ point density is twice the vortex density. Finally, for vortices, scalar fields $a$ and $b$ have the same polarization, whereas for $\mathrm{C}$ points in vector fields $a$ and $b$ are orthogonally polarized.

These essential preliminaries completed, we turn now to our results for speckled speckle in what may be called the perturbation regime, i.e. the regime in which $I_{b} / I_{a} \ll$ 1. Results for other regimes will be reported on separately.

In Fig. 1, which shows scalar fields, we compare normal speckle $\left(I_{b}=0\right)$ with speckled speckle $\left(I_{b}>0\right)$. As can be seen, the vortices of speckled speckle are found only in regions surrounding the $a$ field vortices shown in Fig. 1(b). The reason for this is that at a vortex the total, i.e. composite, field amplitude must vanish. But because $I_{b} \ll I_{a}$, the destructive interference between the $a$ and $b$ fields required for vortex generation can occur only in regions where $I_{a}=I_{b}$, i.e. where $I_{a}$ is very small. This leads to isolated giant clusters of speckled speckle vortices near the positions of the $a$ field vortices. Such a cluster, containing 29 positive and 28 negative vortices is shown in Fig. 1(e). As shown in Figs. 1(f) and 1(g), vortices are absent in other regions.

An anomalous feature of speckled speckle phase maps is a granularity not seen in normal speckle phase maps; not only the intensity, but also the phase of speckled speckle is speckled. This granularity, which is present throughout the phase map, and which can be seen most easily in Fig. 1 ( $(\mathrm{g})$, corresponds to large numbers of phase maxima and minima, i.e. phase extrema.

In normal speckle phase maps vortices $V$ always outnumber extrema $E$ 6, 14]. For a single Gaussian $E / V=$ 0.414 , for a single disk $E / V=0.0887$, and for a single ring $E / V=0$ [14. $E / V$ can be expressed in terms
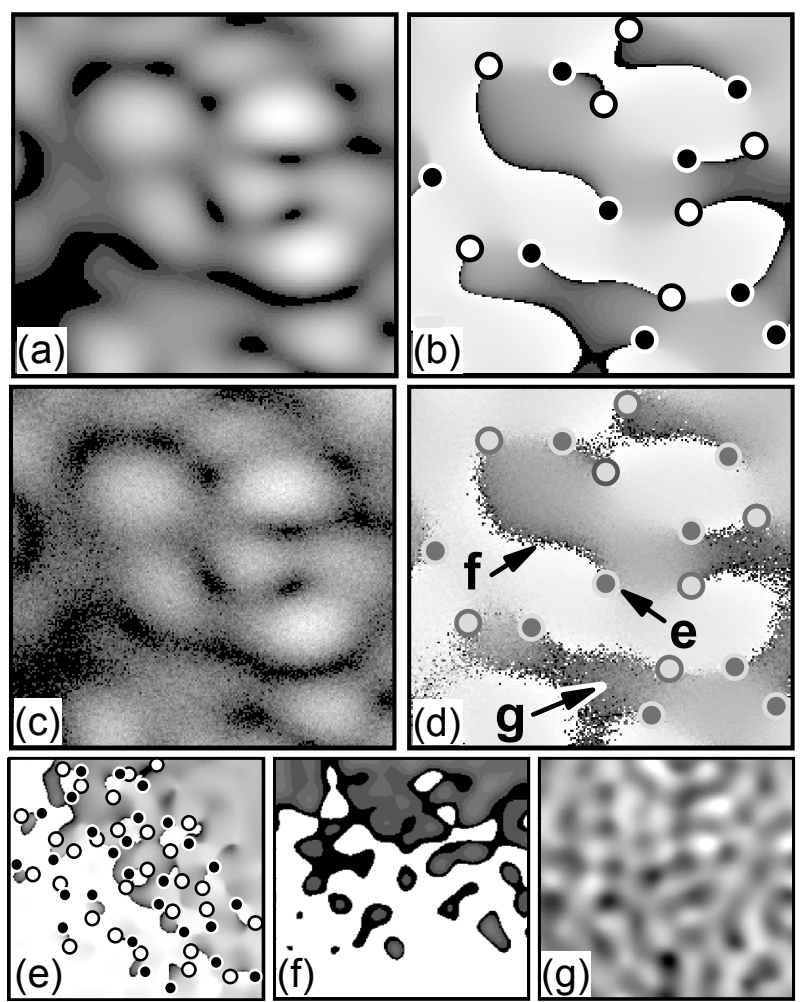

Fig. 1. Scalar speckle. Shown are simulations for disks with $a=1, b=100$. In (a) and (b) $I_{b}=0$, which yields familiar single $\Lambda$ speckle with $\Lambda \sim 1$. In (c) - $(g) I_{b} / I_{a}=$ $2 \cdot 10^{-6}(K=0.02)$, which yields speckled speckle with $\Lambda_{a} \sim 1, \Lambda_{b} \sim 0.01$. (a) Intensity of single $\Lambda$ speckle. (b) Phase map corresponding to (a). (c) Intensity of speckled speckle. (d) Phase map corresponding to (c). (e) - (f) Forty-fold enlargements of the regions in (d) pointed to by the arrows labeled e, f, and g. Here and throughout intensity (phase) is coded increasing (0 to $2 \pi$ ) black to white, and positive (negative) vortices are shown by filled white (black) circles with black (white) rims.

of the moments $M_{n}, n=2,4$, of the source function $S$ [14. For speckled speckle we obtain for these moments $M_{n}=C_{n}\left(a^{n}+K b^{n}\right) /(1+K)$, where for two Gaussians $(2 \mathrm{G}), C_{n}=2^{n}(n / 2)$ !, for two disks (2D), $C_{n}=2 /(n+2)$, and for two rings $(2 \mathrm{R}), C_{n}=1$. Writing $\rho=a / b$, when $K=\rho^{2}, E / V$ attains a maximum value that for small $\rho$ is given by

$$
(E / V)_{\max }=g\left[(3)^{1 / 2} \rho^{2}\right]^{-1},
$$

where for $2 \mathrm{G}, g=1 / 2$, for $2 \mathrm{D}, g=1 / 3$, and for $2 \mathrm{R}$, $g=1 / 4$. In principle $(E / V)_{\max }$ can be made arbitrarily large; for the parameters $a$ and $b$ in Fig. 1 we have for $2 \mathrm{G}, 2 \mathrm{D}$, and $2 \mathrm{R}$, respectively, $(E / V)_{\max }=2887,1924$, and 1443 , corresponding to enhancements over normal speckle by factors of $\sim 7,000, \sim 22,000$, and $\infty$.

$\mathrm{C}$ points show similar anomalies. At a $\mathrm{C}$ point $\left|E_{x}\right|=$ $\left|E_{y}\right|$, so also speckled speckle $\mathrm{C}$ points can be found only in dark regions of the a field, here $E_{x}$. In Fig. 2 we show 

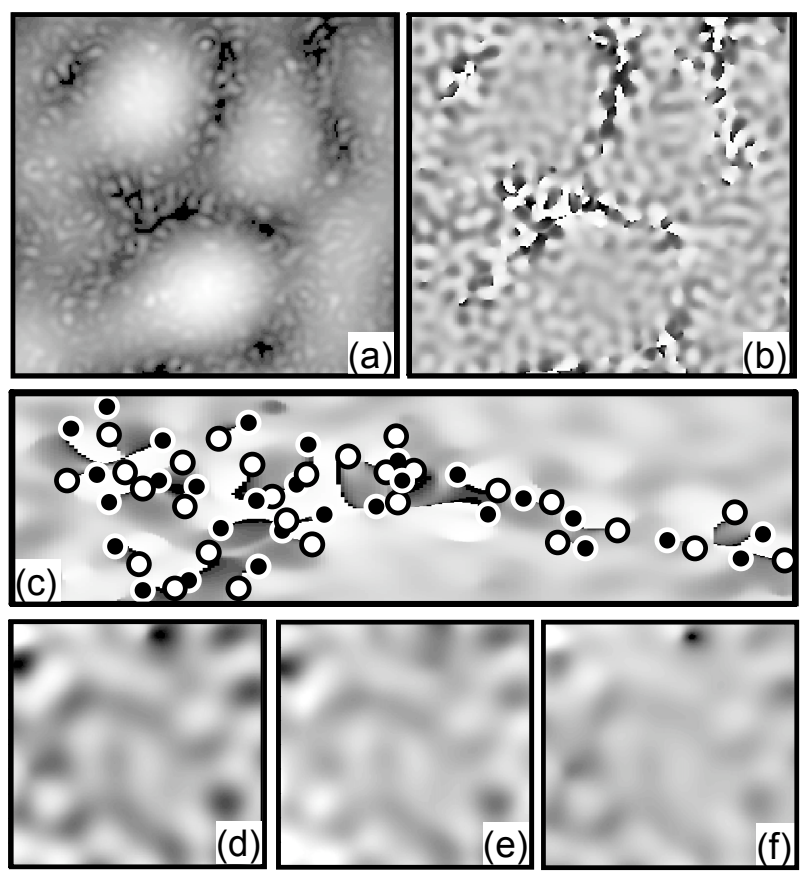

Fig. 2. Vector field speckled speckle. Shown are simulations for disks with $\mathrm{a}=1, \mathrm{~b}=10, I_{b} / I_{a}=3 \cdot 10^{-3}$ $(K=0.3)$. (a) Intensity. (b) Phase $\Phi_{12}$ of complex Stokes field $S_{12}$. (c) Enlargement of the black-white band on the horizontal centerline in (b). (d) - (f) Enlargement of the region beneath the black-white band in (b). (d) Phase $\Phi_{12}$ of $S_{12}$. (e) Phase $-\Phi_{R}$ of $E_{R}$. (f) Phase $\Phi_{L}$ of $E_{L}$.

speckled speckle $\mathrm{C}$ points for a value for $I_{b} / I_{a}$ that is much larger than the corresponding value in Fig. 1. Now $\mathrm{C}$ points are no longer confined to the darkest regions near $a$ field vortices, but can populate dark areas between $a$ field speckle spots, typically forming chains that tend to surround these spots. An example of such a chain, which contains 28 positive and 29 negative $\mathrm{C}$ points, is shown in Fig. 2(c).

Also speckled speckle $\mathrm{C}$ point phase maps contain numerous extrema that are absent in normal speckle $\mathrm{C}$ point maps: in obvious notation $E / C=\frac{1}{2} E / V$. This result follows from the fact that, as shown below, the density of phase extrema is essentially the same in both scalar and vector speckled speckle, whereas the density of $\mathrm{C}$ points is twice the vortex density [12, 13, 22].

From Eqs. (3) we have $E_{R}=E_{x}\left(1-i E_{y} / E_{x}\right)$ $\approx E_{x} \exp \left(-i E_{y /} E_{x}\right), \quad E_{L}=E_{x}\left(1+i E_{y /} E_{x}\right) \approx$ $E_{x} \exp \left(i E_{y} / E_{x}\right)$, where in the approximate equalities we have used the fact that $E_{x}$ is the strong $a$ field, and $E_{y}$ is the weak $b$ field, so that $E_{y /} E_{x}$ is small. Writing $S_{12}=$ $A_{12} \exp \left(i \Phi_{12}\right), E_{x}=A_{x} \exp \left(i \varphi_{x}\right), E_{y}=A_{y} \exp \left(i \varphi_{y}\right)$, $E_{R}=A_{R} \exp \left(i \Phi_{R}\right)$, and $E_{L}=A_{L} \exp \left(i \Phi_{L}\right)$, and using Eqs. (3), we have from Eqs. (2) $\Phi_{12}=\Phi_{L}-\Phi_{R} \approx 2\left(\Phi_{L}-\right.$ $\left.\varphi_{x}\right) \approx-2\left(\Phi_{R}-\varphi_{x}\right)$. But because $\Lambda_{x}=\Lambda_{a} \sim 1 / a$ is large, except close to its sparse vortices $\varphi_{x}$ varies slowly com- pared to $\Phi_{12}, \Phi_{R}$, and $\Phi_{L}$, for which $\Lambda_{12} \approx \Lambda_{R} \approx \Lambda_{L} \sim 1 / b$. Thus except close to its sparse vortices $\varphi_{x}$ can be taken to be nearly constant, and so the phase field $\Phi_{12}$ of vector speckled speckle, and the phase fields $\Phi_{L}$ and $-\Phi_{R}$ of scalar speckled speckle, have very nearly the same set of extrema. This unusual property of speckled speckle is verified in Figs. 2(d)-2(f).

In summary, we have shown that in the perturbation regime studied here the singularities of scalar and vector speckled speckle, and the critical points associated with these singularities, have highly interesting, anomalous spatial arrangements and statistics. Singularity screening, correlations, and other properties of these unusual fields in different parameter regimes are also likely to exhibit unusual new phenomena of special interest.

Email addresses: I. Freund, freund@mail.biu.ac.il; D. A. Kessler, kessler@dave.ph.biu.ac.il.

\section{Acknowledgements}

D. A. Kessler acknowledges the support of the Israel Science Foundation.

\section{References}

1. 1. J. W. Goodman, Speckle Phenomen In Optics (Roberts \& Co., Englewood, Colorado, 2007).

2. M. Berry, "Disruption of wave-fronts: statistics of dislocations in incoherent Gaussian random waves," J. Phys. A. 11, 27-37 (1978).

3. B. I. Halperin, "Statistical mechanics of topological defects," in Physics of Defects, R. Balian, M Kleman, and J.-P. Poirier, eds. (North-Holland, Amsterdam, 1981), pp. 814-857.

4. N. B. Baranova, B. Ya Zel'dovich, A. V. Mamaev, N. Pilipetskii and V. V. Shkukov, "Dislocations of the wave-front of a speckle-inhomogeneous field (theory and experiment)," JETP Lett. 33, 195-199 (1981).

5. F. Liu and G. F. Mazenko, "Defect-defect correlation in the dynamics of first-order phase transitions," Phys. Rev. B 46, 5963-5971 (1992).

6. I. Freund, "Saddles, singularities, and extrema in random phase fields," Phys. Rev. E 52, 2348-2360 (1995).

7. B. W. Roberts, E, Bodenschatz, and J. P. Sethna, "A bound on the decay of defect-defect correlation functions in two-dimensional complex order parameter equations," Physica D 99, 252-268 (1996).

8. I. Freund and D. A. Kesler, "Phase autocorrelation of random fields," Opt. Commu. 124, 321-332 (1996).

9. I. Freund, "' 1001 ' correlations in random wave fields," Waves in Random Media 8, 119-158 (1998).

10. I. Freund and M. Wilkinson, "Critical-point screening in random wave fields," J. Opt. Soc. Am. A 15, 2892-2902 (1998). 
11. M. V. Berry and M. R. Dennis, "Phase singularities in isotropic random waves," Proc. Roy. Soc. London A 456, 2059-2079 (2000).

12. M. V. Berry and M. R. Dennis, "Polarization singularities in isotropic random vector waves," Proc. Roy. Soc. Lond. A 457, 141-155 (2001).

13. M. R. Dennis, "Polarization singularities in paraxial vector fields: morphology and statistics," Opt. Commun. 213, 201-221 (2002).

14. M. R. Dennis, "Phase critical point densities in planar isotropic random waves," J. Phys. A: Math. Gen. 34, L297-L303 (2003).

15. M. S. Soskin, V. Denisenko, and I. Freund, "Optical polarization singularities and ellipric stationary points," Opt. Lett. 28, 1475-1477 (2003).

16. G. Foltin, "Signed zeros of Gaussian vector fields - density, correlation functions, and curvature," J. Phys. A: Math. Gen. 36, 1729-1741 (2003).

17. M. R. Dennis, "Correlations and screening of topological charges in Gaussian random fields," J. Phys. A: Math. Gen. 36, 6611-6628 (2003).
18. M. Wilkinson, "Screening of charged singularities of random fields," J. Phys. A: Math. Gen. 37, 67636771 (2004).

19. M. Soskin, V. Denisenko, and R. Egorov, "Topological networks of paraxial ellipse speckle fields," J. Opt. A 6, S281-S287 (2004).

20. R. I. Egorov, V. G. Denisenko, and M. S. Soskin, "Topological response of inhomogeneous, elliptically polarized, light fields to controlled anisotropic perturbations," JETP Lett. 81, 375-377 (2005).

21. For other applications of this term see 1] Sect. 3.6.

22. J. F. Nye, Natural Focusing and Fine Structure of Light (IOP Publ., Bristol, 1999).

23. A.I. Konukhov and L.A. Melnikov, "Optical vortices in a vector field: the general definition based on the analogy with topological solitons in a $2 \mathrm{D}$ ferromagnet, and examples from the transverse polarization patterns of lasers," J. Opt. B 3, S139-S144 (2001).

24. I. Freund, "Poincaré vortices," Opt. Lett. 26, 19961998 (2001). 